whose myeloma is refractory to treatment might still recover their renal function. Moreover, cyclized peptides such as the one described can also help patients with monoclonal gammopathy-related kidney diseases. Many of these patients have nonmalignant lymphoproliferative or plasma cell disorder that would not require treatment if not for the renal complication (10). Therapeutic strategies that target the monoclonal protein rather than the plasma cell are extremely attractive because they would avoid the use of toxic chemotherapy in those patients who do not have a malignancy condition. This strategy could be quite useful in patients with AL amyloidosis who may be too frail to go through chemotherapy. The potential treatment opportunities of this concept represent new and less toxic treatments for the entire range of plasma cell diseases.

Address correspondence to: Nelson Leung, 200 First Street SW, Rochester, Minnesota 55905, USA. Phone: 507.266.7083; Fax: 507.266.7891; E-mail: Leung.nelson@ mayo.edu.
1. Steensma DP, Kyle RA. A history of the kidney in plasma cell disorders. Contrib Nephrol. 2007;153:5-24.

2. Sanders PW. Pathogenesis and treatment of myeloma kidney. J Lab Clin Med. 1994;124(4):484-488.

3. Dimopoulos MA, et al. Renal impairment in patients with multiple myeloma: a consensus statement on behalf of the International Myeloma Working Group. J Clin Oncol. 2010;28(33):4976-4984.

4. Bence Jones $\mathrm{H}$. On a new substance occurring in the urine of a patient with mollities ossium. Phil Trans R Soc London. 1848;138:55-62.

5. Woodruff R, Sweet B. Multiple myeloma with massive Bence Jones proteinuria and preservation of renal function. Aust NZ J Med. 1977;7(1):60-62.

6. Sanders PW, Booker BB, Bishop JB, Cheung HC. Mechanisms of intranephronal proteinaceous cast formation by low molecular weight proteins. J Clin Invest. 1990;85(2):570-576.

7. Ying WZ, Sanders PW. Mapping the binding domain of immunoglobulin light chains for Tamm-Horsfall protein. Am J Pathol. 2001;158(5):1859-1866.

8. Sanders PW, Booker BB. Pathobiology of cast nephropathy from human Bence Jones proteins. J Clin Invest. 1992;89(2):630-639.

9. Ying WZ, Allen CE, Curtis LM, Aaron KJ, Sanders PW. Mechanism and prevention of acute kidney injury from cast nephropathy in a rodent model. J Clin Invest. 2012;122(5):1777-1785.

10. Leung N, Rajkumar SV. Renal manifestations of plasma cell disorders. Am J Kidney Dis. 2007; 50(1):155-165

11. Ying WZ, Wang PX, Aaron KJ, Basnayake K, Sanders PW. Immunoglobulin light chains activate nuclear factor-kappaB in renal epithelial cells through a Src-dependent mechanism. Blood. 2011;
117(4):1301-1307.

12. Knudsen LM, Hjorth M, Hippe E. Renal failure in multiple myeloma: reversibility and impact on the prognosis. Nordic Myeloma Study Group. Eur J Haematol. 2000;65(3):175-181.

13. Blade J, et al. Renal failure in multiple myeloma: presenting features and predictors of outcome in 94 patients from a single institution. Arch Intern Med. 1998;158(17):1889-1893.

14. Hutchison CA, et al. Early reduction of serumfree light chains associates with renal recovery in myeloma kidney. J Am Soc Nephrol. 2011; 22(6):1129-1136

15. Leung $\mathrm{N}$, et al. Improvement of cast nephropathy with plasma exchange depends on the diagnosis and on reduction of serum free light chains. Kidney Int. 2008;73(11):1282-1288.

16. Burnette BL, Leung N, Rajkumar SV. Renal improvement in myeloma with bortezomib plus plasma exchange. N Engl J Med. 2011;364(24):2365-2366.

17. Hutchison CA, et al. Efficient removal of immunoglobulin free light chains by hemodialysis for multiple myeloma: in vitro and in vivo studies. J Am Soc Nephrol. 2007;18(3):886-895.

18. Barlogie B, et al. Long-term follow-up of autotransplantation trials for multiple myeloma: update of protocols conducted by the intergroupe francophone du myelome, southwest oncology group, and university of arkansas for medical sciences. J Clin Oncol. 2010;28(7):1209-1214.

19. Arimura A, Li M, Batuman V. Potential protective action of pituitary adenylate cyclase-activating polypeptide (PACAP38) on in vitro and in vivo models of myeloma kidney injury. Blood. 2006;107(2):661-668

\title{
NADPH oxidase regulates efficacy of vaccination in aspergillosis
}

\author{
George S. Deepe Jr.
}

Division of Infectious Diseases, University of Cincinnati College of Medicine, and the Veterans Affairs Hospital, Cincinnati, Ohio, USA.

\begin{abstract}
Invasive aspergillosis is often a consequence of immune suppression, and accumulating evidence points to a role for adaptive immunity. Hence, it may be possible to manipulate the adaptive immune system to enhance protective immunity in at-risk individuals. In this issue of the JCI, De Luca and colleagues describe the ontogeny of adaptive immune responses to murine aspergillosis infection in relation to vaccination. Their thought-provoking findings reveal the complexities of vaccine-induced immunity and could be used to improve vaccine efficacy.
\end{abstract}

Aspergillus spp constitute an omnipresent mold, found in air, water, and soil. Conidia - spores that are released from hyphae - are inhaled daily by humans. The major variable in human exposure is the number of particles, and this value may be low indoors and strikingly high in areas that

Conflict of interest: The author has declared that no conflict of interest exists.

Citation for this article: J Clin Invest. 2012; 122(5):1608-1611. doi:10.1172/JCI63417. contain decaying matter. The species of Aspergillus that cause invasive infection are limited, but these share an important trait: the ability to grow at $37^{\circ} \mathrm{C}$, human body temperature. Among those that cause invasive disease in humans, A. fumigatus is the most prevalent followed, by $A$. flavus $(1,2)$.

Despite constant contact with conidia, the immunocompetent host rarely develops invasive disease, largely because immune defenses in lungs are highly effective. Infec- tion is initiated when host resistance is dampened, and spores convert into hyphae that disseminate through the vascular system. Several risk factors predispose individuals to infection with Aspergillus. Chief among them are the use of glucocorticoids to treat inflammatory disorders, severe neutropenia induced by cytotoxic agents, or, less frequently, the use of TNF- $\alpha$ antagonists. The risk of disease acquisition escalates in parallel with the length of glucocorticoid administration or the duration of neutropenia (1). Qualitative defects in neutrophil function also increase the probability of acquiring disease. One of the more common disorders associated with dysfunctional phagocytes is chronic granulomatous disease (CGD), an inherited disease in which the fundamental genetic defect is in the assembly of the NADPH oxidase (3). Since the leukocytes from these patients fail to mount a vigor- 


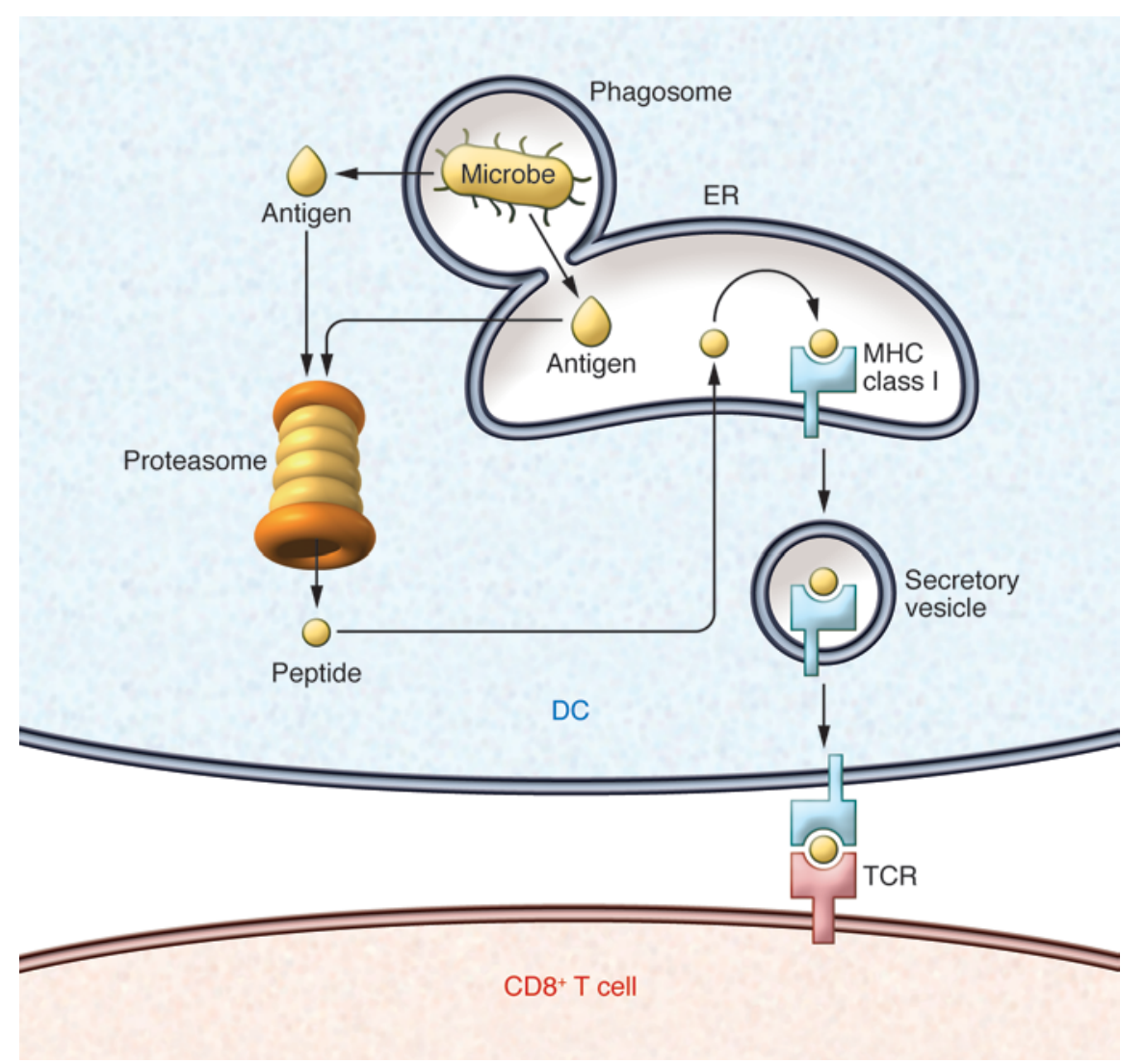

ous respiratory burst, their phagocytes are impaired in their ability to combat numerous pathogens including bacteria and Aspergillus spp. Despite many advances in both early diagnosis and antifungal agents, the attributed mortality for all patients still ranges from $40 \%$ to $70 \%$ (2).

\section{Cellular immunity to Aspergillus}

Although innate immunity appears to be the principal host resistance mechanism, there is mounting evidence that cellular immunity also is crucial. Exposure to A. fumigatus

\section{Figure 2}

$\mathrm{CD}^{+}$and $\mathrm{CD}^{+} \mathrm{T}$ cell recognition of Aspergillus antigens. (A) Soluble antigens such as Pep1, when admixed with soluble antigen, enter the MHC class II pathway whether NADPH oxidase is present or not and promote the development of protective CD4+ cells. (B) On the other hand, particulate immunogens such as conidia enter the MHC class I pathway but only activate CD8+ cells in the presence of NADPH oxidase. The lack of this oxidase, as in CGD, is associated with a failure to induce autophagy and endosomal alkalinization. This defect results in absent activation of $\mathrm{CD}^{+}$cells. spores induces the differentiation of T cells into Th1, Th2, and Th17 cells. The former are pivotal for generation of protective immunity to invasive aspergillosis, whereas Th2 cells are central to the pathogenesis of allergic bronchopulmonary aspergillosis, and they blunt Th1-mediated immunity in invasive infection. Th17 cells promote immunity, but apparently not with the same vigor as Th1 (4-7). Transfer of Th1biased $\mathrm{CD} 4^{+}$cells ameliorates the severity of invasive infection, suggesting that they are the principal mediators of resistance $(8,9)$.
A

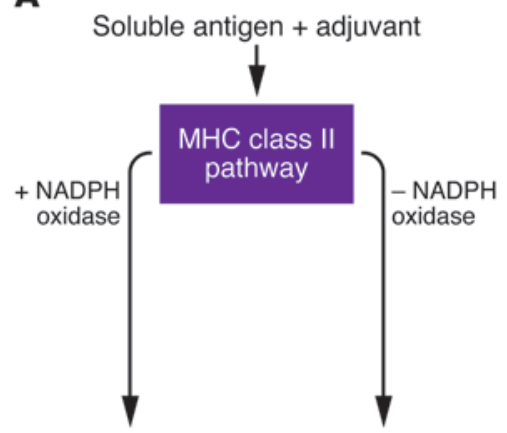

$\mathrm{CD}^{+} \mathrm{T}$ cell activation

\section{Figure 1}

Cross-presentation pathway by antigen-presenting cells. DCs or macrophages engulf antigens into phagosomes. The phagosomes may fuse with endoplasmic reticulum, and antigens from the particle are directed to the proteasome where they are digested; cognate peptides with the appropriate linear amino sequence then bind to MHC I. This complex moves to the surface of an antigen-presenting cell and is subsequently engaged by a $\mathrm{CD}^{+} \mathrm{T}$ cell. An alternative pathway is that antigens "leak" or escape from the phagosome and enter the cytosol in the absence of fusion of phagosome/endoplasmic reticulum; the antigens in the cytosol are digested by the proteasome, bind to $\mathrm{MHC} \mathrm{I}$, and traffic to the cell surface.
The differentiation of $\mathrm{CD}^{+}{ }^{+} \mathrm{T}$ cells into one of the aforementioned families is shaped by inflammatory monocytes and Dectin-1 (4, 10). Less well appreciated is the influence of $\mathrm{CD}^{+} \mathrm{T}$ cells on the host response. Antigen-reactive $\mathrm{CD}^{+} \mathrm{T}$ cells can be elicited by Aspergillus antigens, and protection relies on recognition of fungal RNA by TLR3 (11, 12). That $\mathrm{T}$ cells exert a strong influence on the host response has major implications not only for the immunopathogenesis but also for the design of prophylactic or therapeutic vaccines for this pathogen.
B

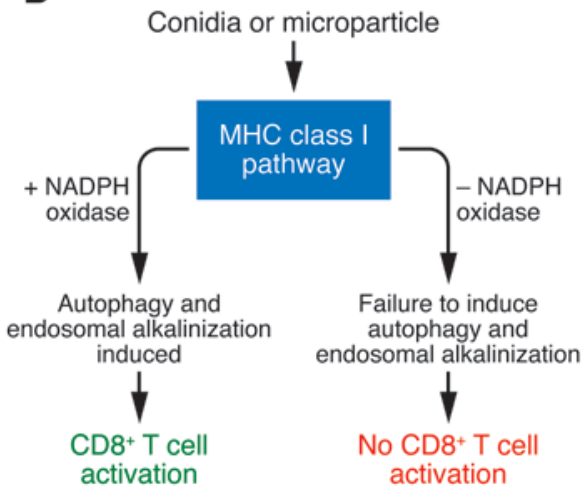


In this issue of the JCI, De Luca et al. (13) provide vital new information regarding the contribution of $\mathrm{CD}^{+}$and $\mathrm{CD}^{+} \mathrm{T}$ cells in host defenses against this devastating fungal pathogen. In this creative work, they use the $p 47^{\text {phox }} /-$ model of CGD, in which the mice have defective NADPH oxidase, to decipher the antigen-presenting pathways in DCs that prompt $\mathrm{CD}^{+}$and $\mathrm{CD}^{+} \mathrm{T}$ cell activation. The authors report that antigen presentation by DCs that lack NADPH oxidase fails to prompt activation of $\mathrm{CD}^{+} \mathrm{T}$ cells.

\section{Divergence of antigen presentation pathways in vaccine-induced immunity to Aspergillus}

Unlike in other studies that have examined the T cell response to Aspergillus, De Luca and colleagues employed a model in which animals are immunized either with live conidia or with one of several recombinant antigens. They found that in wildtype mice, $\mathrm{CD}^{+} \mathrm{T}$ cells are essential for protection upon vaccination with conidia, whereas immunization with soluble Aspergillus proteins admixed with $\mathrm{CPG}$ confers protection in a $\mathrm{CD}^{+} \mathrm{T}$ cell-dependent manner (13). The finding that $\mathrm{CD}^{+} \mathrm{T}$ cells are the mediators of protection following immunization with conidia is somewhat surprising in light of previous work reporting that exposure to these fungal elements in primary infection induces a pronounced $\mathrm{CD}^{+} \mathrm{T}$ cell differentiation (4).

Conidia are engulfed into phagosomes, and no evidence exists that they escape into the cytosol, indicating that cross-presentation by DCs is the mechanism whereby MHC class I gains access to peptides from conidia (Figure 1 and refs. 14-16). Although cross-presentation is a property of both DCs and macrophages, De Luca et al. report that lung macrophages lack the capability of cross-presentation; however, this observation was not expanded upon. The efficacy of vaccination required the presence of IFN- $\gamma$ and to a lesser extent IL-17; these results mimic those reported for primary infection (5). In striking contrast, De Luca and colleagues found that $p 47^{\text {phox- }} /-$ mice develop immunity only when immunized with the soluble antigens, indicating that processing of conidia in the cross-presentation pathway is impaired when DCs lack NADPH oxidase. This finding is not a consequence of an intrinsic dysfunction of $\mathrm{CD}^{+}$cells in these mice, which have been reported to have an elevation in the number of these cells (17).

\section{Pattern recognition receptors dictate} the antigen presentation pathways

The shaping of the host response whether by direct invasion of a pathogen or by a vaccine requires the engagement of pattern recognition receptors (PRRs). These molecules are influential in determining the differentiation of $\mathrm{T}$ cells. Recognition of conidia by $\mathrm{CD}^{+} \mathrm{T}$ cells required TLR 3 and TIR domain-containing adapter-inducing interferon- $\beta$ (TRIF). On the other hand, MyD88 and TLR 6 were necessary for the action of one of the soluble antigens, and other soluble antigens had distinct TLR requirements for driving $\mathrm{CD}^{+} \mathrm{T}$ cell activation (13). This work highlights the complexity of antigen recognition at the level of PRRs.

\section{The intracellular highway for conidia and soluble antigens}

$\mathrm{T}$ cells recognize small peptide epitopes from antigens in the context of the cognate MHC. Proper trafficking of particulate or soluble antigens is a key step in the optimal generation of the epitopes that drive $\mathrm{T}$ cell activation. De Luca et al. demonstrated that protective soluble antigens engaged the mannose receptor and occupied organelles that contained Rab GTPases (13). The importance of the mannose receptor for soluble antigens is not surprising, given that the authors employed glycosylated antigens. Indeed, removal of the carbohydrate moieties abolished their protective activity, emphasizing the utility of glycosylation on immunogens.

De Luca et al. describe an orderly spatial and temporal sequence as the antigens navigated through the DC (Figure 2). Conidia trafficked to the late endosomes and subsequently to Rab $14^{+}$compartments, whereas soluble antigen never occupied these compartments. Autophagy and endosomal alkalinization were fundamental for directing conidia to the Rab14-containing organelles and for cross-presentation. In the absence of phox 47, conidia do not reach $\mathrm{Rab} 14^{+}$compartments, and cells displayed diminished autophagy. Restoration of autophagy enabled DCs to cross-present conidial antigens to $\mathrm{CD} 8^{+} \mathrm{T}$ cells (13). These results raise the possibility that antigens from conidia that have been digested by the intracellular machinery of DCs are captured within autophagosomes and processed for class I MHC export $(15,18)$.

\section{Clinical implications}

De Luca et al. raise a number of issues that are germane to clinical medicine. Their findings clearly point to a defective CD8 ${ }^{+}$
$T$ cell response in mice that lack NADPH oxidase and suggest an underlying mechanism that explains the defect. One concern is the hierarchy of protective immunity conferred by $\mathrm{T}$ cells in aspergillosis. Are $\mathrm{CD}^{+} \mathrm{T}$ cells subservient to $\mathrm{CD} 4^{+} \mathrm{T}$ cells? The present article would suggest that this might not be the case, but more definitive proof will need to be accumulated. It is also unclear whether these findings will translate to human patients. One study published nearly 20 years ago indicated that memory $\mathrm{CD}^{+}$cells were defective in patients with CGD (19), but virtually nothing is known about human $\mathrm{CD}^{+}$cells from CGD patients and cross-presentation pathways. The third issue is that induction of autophagy enhanced cross-presentation and consequently activation of $\mathrm{CD}^{+}$ $T$ cells, suggesting perhaps a more general means to improve antigen recognition. The data presented in this article are congruent with prior reports that rapamycin, an mTOR inhibitor and inducer of autophagy, promotes $\mathrm{CD}^{+} \mathrm{T}$ cell differentiation and memory responses directly or enhances the efficacy of vaccination by acting on DCs (20, $21)$. This agent is principally used to prevent graft rejection. Utilizing it as a means to boost vaccine responses and improve $\mathrm{CD}^{+} \mathrm{T}$ cells responses may be problematic given its side effects, but new compounds that act similarly may help to prevent infections with organisms that remain difficult to cure such as Aspergillus spp.

\section{Acknowledgments}

G.S. Deepe is supported by VA Merit Review Grant 5I01BX000717-02 and by National Institute of Allergy and Infectious Diseases grants AI073337, AI083313, and AI096823.

Address correspondence to: George S. Deepe, University of Cincinnati College of Medicine, Division of Infectious Diseases, 231 Albert Sabin Way, Cincinnati, Ohio 45267-0560, USA. Phone: 513.558.4704; Fax: 513.558.2089; E-mail: george.deepe@uc.edu.

1. Segal BH. Aspergillosis. N Engl J Med. 2009; 360(18):1870-1884

2. Herbrecht R, et al. Voriconazole versus amphotericin B for primary therapy of invasive aspergillosis. NEngl J Med. 2002;347(6):408-415.

3. Segal BH, Romani LR. Invasive aspergillosis in chronic granulomatous disease. Med Mycol. 2009;47(suppl 1):S282-S290.

4. Rivera A, et al. Dectin-1 diversifies Aspergillus fumigatus-specific $\mathrm{T}$ cell responses by inhibiting $\mathrm{T}$ helper type $1 \mathrm{CD} 4 \mathrm{~T}$ cell differentiation. J Exp Med. 2011;208(2):369-381. 
5. Chai LY, et al. Anti-Aspergillus human host defence relies on type $1 \mathrm{~T}$ helper (Th1), rather than type 17 T helper (Th17), cellular immunity. Immunology. 2010;130(1):46-54.

6. Cramer RA, Rivera A, Hohl TM. Immune responses against Aspergillus fumigatus: what have we learned? Curr Opin Infect Dis. 2011;24(4):315-322.

7. Kreindler JL, et al. Vitamin D3 attenuates Th2 responses to Aspergillus fumigatus mounted by CD4 $\mathrm{T}$ cells from cystic fibrosis patients with allergic bronchopulmonary aspergillosis. J Clin Invest. 2010;120(9):3242-3254.

8. Tramsen L, et al. Clinical-scale generation of human anti-Aspergillus $\mathrm{T}$ cells for adoptive immunotherapy. Bone Marrow Transplant. 2009;43(1):13-19.

9. Cenci E, Mencacci A, Bacci A, Bistoni F, Kurup VP, Romani L. T cell vaccination in mice with invasive pulmonary aspergillosis. J Immunol. 2000;165(1):381-388

10. Hohl TM, et al. Inflammatory monocytes facilitate adaptive CD4 $\mathrm{T}$ cell responses during respiratory fungal infection. Cell Host Microbe. 2009;6(5):470-481.

11. Tao J, et al. Aspergillus fumigatus extract differentially regulates antigen-specific $\mathrm{CD}^{+}$and $\mathrm{CD}^{+} \mathrm{T}$ cell responses to promote host immunity. J Leukoc Biol. 2006;80(3):529-537.

12. Carvalho A, et al. TLR3 essentially promotes protective class I-restricted memory $\mathrm{CD}^{+} \mathrm{T}$-cell responses to Aspergillus fumigatus in hematopoietic transplanted patients. Blood. 2012;119(4):967-977.

13. De Luca A, et al. CD4 ${ }^{+} \mathrm{T}$ cell vaccination overcomes defective cross-presentation of fungal antigens in a mouse model of chronic granulomatous disease. J Clin Invest. 2012;122(5):1816-1831.

14. Schnorrer $\mathrm{P}$, et al. The dominant role of $\mathrm{CD}^{+}$ dendritic cells in cross-presentation is not dictated by antigen capture. Proc Natl Acad Sci U S A. 2006;103(28):10729-10734.

15. Chemali M, Radtke K, Desjardins M, English L. Alternative pathways for MHC class I presenta- tion: a new function for autophagy. Cell Mol Life Sci. 2011;68(9):1533-1541.

16. Kurts C, Robinson BW, Knolle PA. Cross-priming in health and disease. Nat Rev Immunol. 2010;10(6):403-414.

17. Donaldson $\mathrm{M}$, et al. p47phox-deficient immune microenvironment signals dysregulate naive T-cell apoptosis. Cell Death Differ. 2009;16(1):125-138

18. Crotzer VL, Blum JS. Autophagy and adaptive immunity. Immunology. 2010;131(1):9-17.

19. Hasui M, et al. Decreased CD4+CD29+ (memory T) cells in patients with chronic granulomatous disease. J Infect Dis. 1993;167(4):983-985.

20. Araki K, et al. mTOR regulates memory CD8 T-cell differentiation. Nature. 2009;460(7251):108-112.

21. Jagannath C, Lindsey DR, Dhandayuthapani S, Xu Y, Hunter RL Jr, Eissa NT. Autophagy enhances the efficacy of BCG vaccine by increasing peptide presentation in mouse dendritic cells. Nat Med. 2009;15(3):267-276.

\title{
Enhancing immune responses to limit chronic immune activation during SIV
}

\author{
Jacob D. Estes \\ AIDS and Cancer Virus Program, SAIC-Frederick Inc., Frederick National Laboratory for Cancer Research, Frederick, Maryland, USA.
}

\begin{abstract}
The persistent immune activation that is typical of HIV-1 and SIV infection results in exhaustion and dysfunction of $T$ and $B$ cells; in $T$ cells, this is marked by increased expression and signaling through the inhibitory receptor programmed death-1 (PD-1). Targeting this exhaustion pathway could result in improved antiviral immune responses, but there have been concerns that it would also lead to increased inflammation and immunopathology. In this issue of the JCI, Dyavar Shetty et al. demonstrate that blocking PD-1 actually reduced proinflammatory responses and improved immunity in the gut of SIV-infected rhesus macaques, suggesting that this might have therapeutic potential to prevent opportunistic infections in HIV-infected patients.
\end{abstract}

Persistent immune activation is a hallmark of HIV-1 infection in humans and pathogenic SIV infection in rhesus macaques (RMs). HIV and SIV infections induce robust, generalized inflammatory responses that begin during acute infection and lead to pathological systemic immune activation, marked by microbial translocation, fibrotic damage of lymphoid tissues, and $\mathrm{CD}^{+}{ }^{+} \mathrm{T}$ cell loss, processes that contribute to pathogenesis and disease progression. Pathological chronic infections with primate immunodeficiency viruses are further characterized by exhausted and dysfunctional $\mathrm{T}$ and $\mathrm{B}$

Conflict of interest: The author has declared that no conflict of interest exists.

Citation for this article: J Clin Invest. 2012; 122(5):1611-1614. doi:10.1172/JCI63389. cell antiviral immune responses $(1,2)$ that contribute to the inability of the host to eliminate the persistent viral pathogen. The exhaustion of virus-specific $\mathrm{T}$ cells in chronically persistent virus infections is not unique to lentivirus infections, as this phenomenon was first demonstrated in the murine lymphocytic choriomeningitis virus (LCMV) model $(3,4)$, and subsequently virus-specific $\mathrm{T}$ cell exhaustion was demonstrated in HIV, HBV, and HCV infections of humans (5-8) and SIV infection of RMs $(9,10)$.

\section{Can preventing exhaustion improve immune responses?}

Exhausted $\mathrm{T}$ cells are characterized by increased expression of the inhibitory receptor programmed death-1 (PD-1), and in the LCMV model, blockade of the
PD-1 pathway restored virus-specific $T$ cell function, leading to viral clearance (11). While blockade of PD-1 enhances HIV- and SIV-specific T cell cytokine production and proliferation in vitro $(9,10)$, initially there was skepticism that this method would restore immunological function in the pathogenic RM SIV model in vivo (12). This skepticism was based on the premise that if in vivo blockade of the PD-1 inhibitory receptor in chronic SIV infection could result in enhanced antiviral $\mathrm{T}$ and $\mathrm{B}$ cell responses, it could also potentially exacerbate bystander $\mathrm{T}$ cell activation in an already persistent inflammatory setting, potentially leading to accelerated disease progression.

Studies using anti-PD-1 antibody in chronically SIV-infected RMs have produced mixed results. Finnefrock et al. showed that a single injection of anti-PD1 antibody produced no change in SIVspecific $T$ cell function or numbers, with a transient increase in viral loads (13), while Velu et al. demonstrated that four repeated administrations of anti-PD-1 antibody resulted in enhanced SIV-specific immunity and prolonged survival (14). In this issue of the JCI, this latter group expands their analysis of anti-PD-1-treated RMs in an attempt to understand the putative mechanism for prolonged survival (15). 